\title{
Empirical Adequacy in the Received View
}

\author{
Sebastian Lutz
}

Preprint: 2013-07-07

\begin{abstract}
I show that the central notion of Constructive Empiricism, empirical adequacy, can be expressed syntactically and specifically in the Received View of the logical empiricists. The formalization shows that the Received View is superior to Constructive Empiricism in the treatment of theories involving unobservable objects or functions from observable to unobservable objects. It also suggests a formalization of 'full empirical informativeness' in Constructive Empiricism.
\end{abstract}

\section{Contents}

\section{Introduction}

2 Two accounts of the relation between theory and observation 3

3 Syntactic empirical adequacy 6

4 Empirical adequacy in a bipartitioned vocabulary $\quad 7$

5 Beyond empirical substructures 9

6 Beyond consistency 11

7 Conclusion $\quad 12$

*Munich Center for Mathematical Philosophy, Ludwig-Maximilians-Universität München. sebastian.lutz@gmx.net. Early versions of this article have been presented at the Lunch Talk of the Theoretical Philosophy Unit at Utrecht University and at the PhDs in Logic conference at Ghent University in 2009. I thank the audiences, the audience at the Twenty-Third Biennial Meeting of the Philosophy of Science Association, and Thomas Müller for helpful discussions. Research for this article was in part supported by the Alexander von Humboldt Foundation. 


\section{Introduction}

The so-called Received View in the philosophy of science (Rv), developed by the logical empiricists and especially by Carnap and Hempel, assumes the formalization of scientific theories in predicate logic and a bipartition of the non-logical vocabulary into observational and theoretical terms. While it was never generally accepted (Richardson 2007, 362-365), it suffered a complete fall from grace (and then some) in the second half of the $20^{\text {th }}$ century (cf. Lutz 2012, $\$ 1$ ). Only recently have there been defenses of Carnap's general philosophical method (Carus 2007, Maher 2007, Lutz 2009, Justus 2012) and of the use of predicate logic or more generally syntactic means for the analysis of scientific theories (Halvorson 2012; 2013, Lutz 2014). The RV itself, however, has so far mainly seen historical defenses (Mormann 2007, Lutz 2012). It is the aim of this article to provide a conceptual defense relative to one of its main competitors, Constructive Empiricism (CE) (van Fraassen 1980). Specifically, I will argue that the relation between theory and observations that is described in the CE-formalism can also be described in the Rv-formalism, while the converse does not hold.

The only previous defense of the RV-formalism relative to CE (Friedman 1982, 276-277) consists of a valuable, but very short outline of an argument and furthermore assumes a restriction to first order logic. Other relative defenses have focused on syntactic approaches in general (Worrall 1984, \$2; Turney 1990). Turney provides the technically most elaborate defense. After proving the equivalence of van Fraassen's notion of embedding and his syntactic notion of implanting (under specific assumptions), he concludes (Turney 1990, 448-449):

I agree with van Fraassen, that the positivist account does not yield good definitions for empirical equivalence and empirical adequacy. However, I do not agree with his claim that this is because the positivist account is syntactic, while his is semantic. [...] The relevant distinction here $[\ldots]$ is between two ways of linking theory and observation: Correspondence rules versus embedding/implanting.

Turney provides a detailed argument for the equivalence of his syntactic and van Fraassen's semantic account, but that van Fraassen's embeddings are an improvement over correspondence rules remains a conjecture.

To disprove Turney's (and van Fraassen's) conjecture, I will provide a definition of the RV-formalism and a definition of the formal core of CE, empirical adequacy. The latter is in conflict with Turney's definition, which I will show to be inadequate $(\$ 2)$. Next, I will provide a syntactic description of empirical adequacy $\left(\$_{3}\right)$, which forms the basis for a description of empirical adequacy on the basis of correspondence rules $\left(\mathbb{S}_{4}\right)$. This, then, shows that whatever the relation between theory and observations is, it can be described in the CE-formalism only if it can be described in the Rv-formalism. The converse does not hold without significantly rewriting scientific theories, however, and I show that this puts CE at a disadvantage $\left(\$_{5}\right)$. Finally, I give a precise description of a problem pointed 
out by Worrall $\left(1984, \mathbb{S}_{3}\right)$ and show how it can be solved in $\mathrm{CE}$ by taking a cue from the Rv.

\section{Two accounts of the relation between theory and ob- servation}

Like the CE-formalism, the Rv-formalism provides a framework for analyzing scientific theories and their relation to observations. The most important reconstructions of theories and most important tools developed in the RV assume a formalization of theories in higher order predicate logic with a vocabulary $\mathscr{V}=\left\{P_{i}, F_{j}, c_{k}: i \in I, j \in J, k \in K\right\}$ of distinct symbols, where each $c_{k}$ is an object symbol and each $P_{i}$ and $F_{j}$ is a relation symbol or, respectively, function symbol of a specific arity and type. Formal model theory is used for the definition of logical entailment (Lutz 2012, 83). What distinguishes the RV from syntactic approaches in general is the assumed bipartition of the non-logical vocabulary $\mathscr{V}$ of a theory into a set $\mathscr{O}$ of observation terms and a set $\mathscr{T}$ of theoretical terms. ${ }^{1}$ This bipartition leads to a natural tripartition of the set of sentences into $\mathscr{O}$-sentences, $\mathscr{T}$-sentences, and correspondence rules, the latter of which are sentences containing both $\mathscr{O}$ - and $\mathscr{T}$-terms (Carnap 1966, ch. 24).

Definition 1. An $R V$-theory $\Theta$ is the union of a set $\Pi$ of higher order $\mathscr{T}$-sentences (the postulates of the theory) and a set $\Gamma$ of higher order $\mathscr{V}$-sentences (the correspondence rules).

Sentences in the extended observation language can be of any order. This is, for example, assumed by Carnap's explication of a theory's synthetic component as its Ramsey sentence (Carnap 1963, \$24.D).

Definition 2. Observations are given by a set of higher order $\mathscr{O}$-sentences.

The moniker 'observations' is of course somewhat misleading, but it will be convenient in the following. What is important is that sets of $\mathscr{O}$-sentences are, in a sense, completely interpreted (cf. Carnap 1963, $\$ 24 . A$ ).

In contradistinction to the RV, the CE-formalism relies on model theory. Van Fraassen $(1980,64)$ describes it as follows:

To present a theory is to specify a family of structures, its models; and secondly, to specify certain parts of those models (the empirical substructures) as candidates for the direct representation of observable phenomena.

Furthermore the models of the theory "are describable only up to structural isomorphism" (van Fraassen 2008, 238; cf. 2002, 22). More formally, this can be phrased as follows:

${ }^{1}$ Following standard terminology in the philosophy of science (but unfortunately not in logic), I use 'term' synonymously with 'non-logical symbol'. 
Definition 3. A CE-theory $\left\langle\left\{\mathfrak{T}_{n}\right\}_{n \in N},\left\{\mathbf{E}_{n}\right\}_{n \in N}\right\rangle$ contains a family of structures (the models of the theory) and for each structure $\mathfrak{T}_{n}=\left\langle T_{n}, P_{i}^{\mathfrak{T}_{n}}, F_{j}^{\mathfrak{T}_{n}}\right.$, $\left.c_{k}^{\mathfrak{T}_{n}}\right\rangle_{i \in I_{n}, j \in J_{n}, k \in K_{n}}$ a set $\mathbf{E}_{n}$ of empirical substructures, such that for each $\mathfrak{E} \in \mathbf{E}_{n}$, $\mathfrak{E} \subseteq \mathfrak{T}_{n}{ }^{2}$ With each model, a theory also contains every isomorphic structure and its corresponding ${ }^{3}$ empirical substructures.

Here and in the following, $T_{n}:=\left|\mathfrak{T}_{n}\right|$. As a shorthand, define furthermore $\left\langle\mathfrak{T}_{n}, \mathbf{E}_{n}\right\rangle:=\left\langle\left\{\mathfrak{T}_{n}\right\}_{n \in N},\left\{\mathbf{E}_{n}\right\}_{n \in N}\right\rangle$. Definition 3 uses a standard formalization of the notion of a structure (Chang and Keisler 1990, 19-20; Hodges 1993, 2). Van Fraassen $(1989,366$, n. 4$)$ objects to structures being "yolked to a particular syntax", as in "many standard logic texts", which suggests that he would prefer a definition phrased in the formalism of Bell and Machover $(1977,164)$, who use an index set instead of a vocabulary. This difference is little more than notational, however, since in their definitions of further model theoretic concepts, the index set plays the role of the vocabulary. For examples relevant in the following, compare the definitions of reduct (or "reduction"), isomorphism, and substructure by Bell and Machover (1977, 164-165) and by Chang and Keisler (1990, 20-23), respectively (cf. Lutz 2014, $\$ 3$ ). A reader preferring Bell and Machover's notation will have no problems adapting the following discussion.

Strictly distinguishing between the observable and unobservable objects, van Fraassen $(1980,64)$ suggests describing observable phenomena by structures as well: "The structures which can be described in experimental and measurement reports we can call appearances" (cf. van Fraassen 2008, 286). This suggests

Definition 4. Appearances are given by a set $\mathbf{A}$ of structures such that the domain of each $\mathfrak{A} \in \mathbf{A}$ contains only observable objects. A structure $\mathfrak{A} \in \mathbf{A}$ is an appearance.

Note that the set of appearances does not have to be closed under isomorphism.

In early works van Fraassen $(1970, \$ 3)$ relies on "elementary statements" and a "satisfaction function" to give the relation between theory and observations, but later van Fraassen $(1980,64)$ defines a theory to be "empirically adequate if it has some model such that all appearances are isomorphic to empirical substructures of that model" (cf. van Fraassen 1991, 12).

Definition 5. A CE-theory $\left\langle\mathfrak{T}_{n}, \mathbf{E}_{n}\right\rangle$ is empirically adequate for appearances $\mathbf{A}$ if and only if there is some $n \in N$ such that for every $\mathfrak{A} \in \mathbf{A}$, there is an $\mathfrak{E} \in \mathbf{E}_{n}$ with $\mathfrak{E} \cong \mathfrak{A}$.

\footnotetext{
${ }^{2}$ The notation for $\mathfrak{T}_{n}$ is close to one often used in the philosophy of science, although it is somewhat infelicitous. The precise notation would be $\mathfrak{T}_{n}=\left\langle T_{n},\left\{\left\langle P_{i}, P_{i}^{\mathfrak{T}_{n}}\right\rangle: i \in I\right\}\right.$, etc. $\rangle$

${ }^{3}$ To be precise: If $f: T_{m} \longrightarrow T_{n}$ is an isomorphism between $\mathfrak{T}_{m}$ and $\mathfrak{T}_{n}$, then the set $\mathbf{E}_{n}$ of empirical substructures that corresponds to $\mathbf{E}_{m}$ contains all and only those structures $\mathfrak{E}$ for which there is an $\mathfrak{E}^{\prime} \in \mathbf{E}_{n}$ such that $f$ is an isomorphism between $\mathfrak{E}$ and $\mathfrak{E}^{\prime}$.
} 
Definition 5 defines the empirical adequacy of a theory relative to a set of appearances. In contradistinction, empirical adequacy simpliciter is defined as empirical adequacy for the set of all appearances (cf. Monton and Mohler 2008, $\$ 1.5)$.

Definition 5 relies on the standard notion of isomorphism (e. g., Hodges 1993, 5). Turney $(1990,430)$ notes that his definitions are "loosely based" on Bell and Machover (1977, 161-168), but he defines the isomorphism of two relational structures as follows (Turney 1990, 430-431, my terminology):

Definition 6. Two relational structures $\mathfrak{S}$ and $\mathfrak{S}^{\prime}$ are idiosyncratically isomorphic if and only if $\mathfrak{S}=\left\langle S, P_{1}, P_{2}, \ldots, P_{k}\right\rangle$ and $\mathfrak{S}^{\prime}=\left\langle S^{\prime}, P_{1}^{\prime}, P_{2}^{\prime}, \ldots, P_{k}^{\prime}\right\rangle$ with $n_{i}$-ary relations $P_{i}, P_{i}^{\prime}$ and there are bijections $f: S \longrightarrow S^{\prime}$ and $g:\left\{P_{1}, \ldots, P_{k}\right\} \longrightarrow$ $\left\{P_{1}^{\prime}, \ldots, P_{k}^{\prime}\right\}$ such that $\left\langle u_{1}, \ldots, u_{n_{i}}\right\rangle \in P_{i} \Leftrightarrow\left\langle f\left(u_{1}\right), \ldots, f\left(u_{n_{i}}\right)\right\rangle \in g\left(P_{i}\right)$.

In contradistinction, Bell and Machover $(1977,165)$ effectively assume that the bijection $g$ is determined to map $P_{i}$ to $P_{i}^{\prime}$ for all $1 \leq i \leq k$ (which leads, up to notational change, to the definition given by Hodges (1993)). Clearly, then, more structures are idiosyncratically isomorphic than isomorphic to each othertoo many more, as one can see from the following example loosely inspired by Halvorson $(2012,192)$. Let the CE-theory $\left\langle\mathfrak{T}_{n}, \mathbf{E}_{n}\right\rangle$ contain all $\mathfrak{T}_{n}$ and $\mathbf{E}_{n}$ that fulfill the following conditions: $\mathfrak{T}_{n}$ is a model of $\Theta=\left\{\exists^{>k} x(x=x)\right\} \cup\left\{\forall x\left(P_{k} x \rightarrow\right.\right.$ $\left.\left.P_{i} x\right): 1 \leq i<k\right\}$ with a set of objects $\left\{a_{i}: 1 \leq i \leq k+1\right\} \subseteq T_{n}$ with $a_{i} \neq a_{j}$ for $i \neq j$, and $\mathbf{E}_{n}$ contains the substructures $\mathfrak{E}_{n}^{i}$ of $\mathfrak{T}_{n}$ with domains $E_{n}^{i}=\left\{a_{i}\right\}$. $\left\langle\mathfrak{T}_{n}, \mathbf{E}_{n}\right\rangle$ makes the substantial claim about observable objects that when an object has property $P_{k}$, it also has all properties $P_{1}, \ldots, P_{k-1}$. But in Turney's definition, $\left\langle\mathfrak{T}_{n}, \mathbf{E}_{n}\right\rangle$ is empirically adequate given any class $\mathbf{A}$ of appearances with singleton domains and properties $P_{1}, \ldots, P_{k}$.

Proof. Take any appearance $\mathfrak{A} \in \mathbf{A} .\left\langle\mathfrak{T}_{n}, \mathbf{E}_{n}\right\rangle$ contains the model $\mathfrak{T}_{n}$ with $T_{n}=\mathbb{N}$ and $P_{i}^{\mathfrak{T}_{n}}=\{i+1, \ldots, k+1\}$ for $1 \leq i \leq k$, and empirical substructures $\mathbf{E}_{n}=\left\{\mathfrak{E}_{n}^{i}\right.$ : $1 \leq i \leq k+1\}$ with $E_{n}^{i}=\{i\}$ and $i \in P_{j}^{\mathfrak{E}_{n}^{i}}$ iff $i>j$. In $\mathfrak{A}$, the single object $a \in A$ has some number $l$ of distinct properties: none for $l=0$ and properties $P_{i_{1}}, \ldots, P_{i_{l}}$ for $1 \leq l \leq k$. For each such $l, \mathfrak{A}$ and $\mathfrak{E}_{n}^{l+1}$ are idiosyncratically isomorphic because $f_{l}: A \longrightarrow E_{n}^{l+1}, a \mapsto l+1$, is trivially a bijection, so that $\mathfrak{A}$, $\mathfrak{E}_{n}^{l+1}, f_{l}$ and $g_{l}$ fulfill definition 6 when $g_{l}$ is chosen as follows. For $l=0, g_{l}=\mathrm{id}$, and for $l>0, g_{l}$ is any bijection from $\left\{P_{1}^{\mathfrak{A}}, \ldots, P_{k}^{\mathfrak{A}}\right\}$ to $\left\{P_{1}^{\mathfrak{E}_{n}^{l+1}}, \ldots, P_{k}^{\mathfrak{E}_{n}^{l+1}}\right\}$ with $g_{l}: P_{i_{j}}^{\mathfrak{A}} \mapsto P_{j}^{\mathfrak{E}_{n}^{l+1}}$ for $1 \leq j \leq l$. Hence every appearance in $\mathbf{A}$ is idiosyncratically isomorphic to an empirical substructure of the same model $\mathfrak{T}_{n}$, and therefore $\left\langle\mathfrak{T}_{n}, \mathbf{E}_{n}\right\rangle$ would be empirically adequate given A under Turney's definition.

It is for this reason that Turney's definition is inadequate. 


\section{Syntactic empirical adequacy}

I now want to provide a syntactic description of the notion of a CE-theory and of empirical adequacy. In a CE-theory, each element $\mathfrak{E} \in \mathbf{E}_{n}$ is uniquely determined as the restriction $\mathfrak{T}_{n} \mid E$ of $\mathfrak{T}_{n}$ to $\mathfrak{E}$ 's domain $E$. Thus for each $\mathfrak{T}_{n}, \mathbf{E}_{n}$ is determined by the set $\left\{E: \mathfrak{E} \in \mathbf{E}_{n}\right\}$, and each CE-theory is completely determined by its corresponding family, defined as follows:

Definition 7. Let $\left\langle\mathfrak{T}_{n}, \mathbf{E}_{n}\right\rangle$ be a CE-theory. Then its corresponding family of structures is given by $\left\{\mathfrak{T}_{n}^{*}\right\}_{n \in N}$ with $\mathfrak{T}_{n}^{*}=\left\langle T_{n}, P_{i}^{\mathfrak{T}_{n}}, F_{j}^{\mathfrak{T}_{n}}, c_{k}^{\mathfrak{T}_{n}}, S^{\mathfrak{T}_{n}^{*}}\right\rangle_{i \in I, j \in J, k \in K}$, where $S^{\mathfrak{T}_{n}^{*}}=\left\{E: \mathfrak{E} \in \mathbf{E}_{n}\right\}$.

$\left\{E: \mathfrak{E} \in \mathbf{E}_{n}\right\}$ is thus the extension of a predicate $S$ of higher order, and for any predicate $P, S P$ is the claim that $P$ has an empirical extension. If $\left\langle\mathfrak{T}_{n}, \mathbf{E}_{n}\right\rangle$ is a CEtheory, its corresponding family can be described up to isomorphism by a set $\Theta$ of higher order sentences or a class $\left\{\Theta_{i}: m \in M\right\}$ thereof, where $\left\{\mathfrak{T}_{n}^{*}\right\}_{n \in N}$ is the union of the models of each set $\Theta_{m}$ (Lutz 2014, $\$ 2$ ). ${ }^{4}$ For a class to be necessary, however, $M$ or at least one $\Theta_{m}$ have to be infinite, for otherwise the corresponding family would be the class of models of the single sentence $\bigvee_{m \in M} \wedge \Theta_{m}$. I am not aware of any scientific theory that would require an infinite $M$ or $\Theta_{m}$, and it seems that for most scientific theories, even finite sets of sentences in higher order logic suffice. For the typical mathematical structures needed in physics can be defined by finite sets of higher order sentences (cf. Enderton 2009, $\$ 2, \mathbb{4}$ ). Nonetheless, I will first discuss CE-theories whose corresponding family can be described by a single set of sentences and then discuss the general case.

Each appearance $\mathfrak{A} \in \mathrm{A}$ can be described up to isomorphism by a single set of sentences $\Phi_{\mathfrak{A}}$. Of course, each $\mathfrak{A}$ may have its own distinct domain $A$, so that the sentences in $\Phi_{\mathfrak{A}}$ must be taken as relativized to $A$. Using the symbol ' $E_{\mathfrak{A}}$ ' as name for $A$, this leads to a set $\Phi_{\mathfrak{A}}^{\left(E_{\mathfrak{A}}\right)}$ of relativized sentences. 5 The set of all appearances can then be described as the set of sentences $\left\{\Phi_{\mathfrak{A}}^{\left(E_{\mathfrak{A}}\right)}: \mathfrak{A} \in \mathbf{A}\right\} \cup\left\{S E_{\mathfrak{A}}\right.$ : $\mathfrak{A} \in \mathbf{A}\}$, containing the relativized descriptions of each appearance and for each appearance's domain $E_{\mathfrak{A}}$ the higher order sentence $S E_{\mathfrak{A}}$ stating that $E_{\mathfrak{A}}$ has an empirical extension. Empirical adequacy then becomes semantic consistency:

Claim 1. Let $\left\{\mathfrak{T}_{n}^{*}\right\}_{n \in N}$ be the family corresponding to CE-theory $\left\langle\mathfrak{T}_{n}, \mathbf{E}_{n}\right\rangle$, and let $\Theta$ describe $\left\{\mathfrak{T}_{n}^{*}\right\}_{n \in N}$ up to isomorphism. Let each appearance $\mathfrak{A} \in \mathbf{A}$ be described up to isomorphism by $\Phi_{\mathfrak{A}}$. Then $\left\langle\mathfrak{T}_{n}, \mathbf{E}_{n}\right\rangle$ is empirically adequate for $\mathbf{A}$ if and only if

$$
\Theta \cup\left\{\Phi_{\mathfrak{A}}^{\left(E_{\mathfrak{A}}\right)}: \mathfrak{A} \in \mathbf{A}\right\} \cup\left\{S E_{\mathfrak{A}}: \mathfrak{A} \in \mathbf{A}\right\}
$$

is semantically consistent.

\footnotetext{
${ }^{4}$ If the CE-theory is formalized in the notation of Bell and Machover, the index set can be used as a vocabulary (cf. Lutz $2014, \$_{3}$ ).

${ }^{5}$ The relativization $\sigma^{\left(E_{\mathfrak{A}}\right)}$ of a sentence $\sigma$ consists of the restriction of all quantifiers in $\sigma$ to $E_{\mathfrak{A}}$ (cf. Hodges 1993, theorem 5.1.1). For any set $\Sigma$ of formulas, $\Sigma^{\left(E_{\mathfrak{A}}\right)}$ is the set of the relativizations of the elements of $\Sigma$. Turney (1990, definition 13) calls relativizations 'qualifications'.
} 
Proof. The set (1) is semantically consistent iff it has a model. This is the case iff there is some model $\mathfrak{T}_{n}^{*}$ of $\Theta$ such that for each $\mathfrak{A} \in \mathbf{A}, \mathfrak{T}_{n}^{*} \vDash\left\{S E_{\mathfrak{A}}\right\} \cup \Phi_{\mathfrak{A}}^{\left(E_{\mathfrak{A}}\right)}$. Because $S^{\mathfrak{T}_{m}^{*}}=\left\{E: \mathfrak{E} \in \mathbf{E}_{n}\right\}$, this holds iff $E_{\mathfrak{A}}$ is interpreted by the domain $E$ of some $\mathfrak{E} \in \mathrm{E}_{n}$ such that $\mathfrak{T}_{n}^{*}\left[E_{\mathfrak{A}} / E\right] \vDash \Phi_{\mathfrak{A}}^{\left(E_{\mathfrak{A}}\right)}$. Since $\Phi_{\mathfrak{A}}^{\left(E_{\mathfrak{A}}\right)}$ does not contain the term $S$, this is the case iff $\mathfrak{T}_{n}\left[E_{\mathfrak{A}} / E\right] \vDash \Phi_{\mathfrak{A}}^{\left(E_{\mathfrak{A}}\right)}$. Induction on the complexity of formulas shows that this holds iff $\mathfrak{E}=\mathfrak{T}_{n} \mid E \vDash \Phi_{\mathfrak{A}},{ }^{6}$ and thus, by assumption, iff $\mathfrak{E}$ is isomorphic to $\mathfrak{A}$. Therefore, the set (1) is semantically consistent iff there is some $n \in N$ such that for each $\mathfrak{A} \in \mathbf{A}$, there is an $\mathfrak{E} \in \mathbf{E}_{n}$ with $\mathfrak{E} \cong \mathfrak{A}$.

If a CE-theory $\left\langle\mathfrak{T}_{n}, \mathbf{E}_{n}\right\rangle$ can be described by a set $\left\{\Theta_{m}: m \in M\right\}$ of sets of sentences, claim 1 entails that $\left\langle\mathfrak{T}_{n}, \mathbf{E}_{n}\right\rangle$ is empirically adequate if and only if $\Theta_{m} \cup$ $\left\{\Phi_{\mathfrak{A}}^{\left(E_{\mathfrak{A}}\right)}: \mathfrak{A} \in \mathbf{A}\right\} \cup\left\{S E_{\mathfrak{A}}: \mathfrak{A} \in \mathbf{A}\right\}$ is semantically consistent for at least one $m \in M$. The use of semantic consistency is not forbidden for syntactic approaches, since their core assumption is not that semantic concepts cannot be used at all, but rather that the description of theories has to be syntactical (cf. Lutz 2014, \5).

The syntactic description of empirical adequacy that is given in claim 1 differs from the one given by Turney $(1990,446-447)$ in a number of respects. For example, Turney assumes that a CE-theory is an elementary class that contains only relational structures. The former arguably puts him at odds with van Fraassen $(1989,211)$, while the latter requires elaborate reformulations of most scientific theories, which typically contain constants and functions. Most importantly for the following, Turney provides a syntactic reformulation of each concept of definition 5 , but keeps its overall structure intact. Specifically, he does not provide a single set of sentences that captures both the family of models and the family of sets of empirical substructures of a CE-theory. For this reason, the possibility to capture empirical adequacy in the RV is not obvious in his syntactic description.

\section{Empirical adequacy in a bipartitioned vocabulary}

Given that the empirical adequacy of a theory can be phrased syntactically in a single vocabulary, it is very simple to capture empirical adequacy in the bipartitioned vocabulary of the Rv. For this, one can simply treat the original vocabulary as theoretical $(\mathscr{T})$ and introduce a second, observational term for each theoretical one. Thus, for each $P_{i}$ one can introduce an observational term $P_{i}^{o}$, and analogously for function and constant symbols, leading to an observational vocabulary $\mathscr{O}$. Let $\Phi^{o}$ be the set of sentences that results from exchanging each non-logical constant in the set $\Phi$ of sentences by its observational counterpart. Then the empirical adequacy of a CE-theory can be expressed as the compatibility of an RV-theory with the observations:

\footnotetext{
${ }^{6}$ For a quick plausibility consideration, note that $\mathfrak{T}_{n}\left[E_{\mathfrak{A}} / E\right] \vDash \Phi_{\mathfrak{A}}^{\left(E_{\mathfrak{A}}\right)} \Leftrightarrow \mathfrak{T}_{n}\left[E_{\mathfrak{A}} / E\right] \mid E \vDash$ $\Phi_{\mathfrak{A}}^{\left(E_{\mathfrak{A}}\right)} \Leftrightarrow \mathfrak{T}_{n}\left[E_{\mathfrak{A}} / E\right]\left|E \vDash \Phi_{\mathfrak{A}}^{\left(E_{\mathfrak{A}}\right)} \wedge \forall x E_{\mathfrak{A}} x \Leftrightarrow \mathfrak{E}=\mathfrak{T}_{n}\right| E \vDash \Phi_{\mathfrak{A}}$.
} 
Corollary 2. Let $\left\{\mathfrak{T}_{n}^{*}\right\}_{n \in N}$ be the family corresponding to CE-theory $\left\langle\mathfrak{T}_{n}, \mathbf{E}_{n}\right\rangle$, and let $\Pi$ describe $\left\{\mathfrak{T}_{n}^{*}\right\}_{n \in N}$ up to isomorphism. Let each appearance $\mathfrak{A}$ be described up to isomorphism by $\Phi_{\mathfrak{A}}$. Then $\left\langle\mathfrak{T}_{n}, \mathbf{E}_{n}\right\rangle$ is empirically adequate for $\mathbf{A}$ if and only if

$$
\Pi \cup \Gamma \cup\left\{\left(\Phi_{\mathfrak{A}}^{\left(E_{\mathfrak{A}}\right)}\right)^{o}: \mathfrak{A} \in \mathbf{A}\right\} \cup\left\{S^{o} E_{\mathfrak{A}}^{o}: \mathfrak{A} \in \mathbf{A}\right\}
$$

is semantically consistent, where $\Gamma=\left\{P_{i}^{o}=P_{i}\right\}_{i \in I} \cup\left\{S^{o}=S\right\} \cup\left\{F_{j}^{o}=F_{j}\right\}_{j \in J} \cup\left\{c_{k}^{o}=\right.$ $\left.c_{k}\right\}_{k \in K}$.

Proof. The reducts of the models of $\Pi \cup \Gamma$ to $\mathscr{O}$ are the models of $\Pi$ with their symbols renamed as described above. Hence the corollary follows from claim 1 by a simple renaming of symbols.

Corollary 2 establishes that empirical adequacy is nothing but the compatibility of an RV-theory consisting of theoretical postulates $\Pi$ and correspondence rules $\Gamma$ (definition 1) with observation sentences (definition 2). As claim 1, corollary 2 applies only to CE-theories whose corresponding family can be described by a single set of higher order sentences. Again with the caveat that few if any theories will require a description of their models by a class $\left\{\Pi_{m}: m \in M\right\}$ of sets of sentences, corollary 2 can be generalized for this case: The CE-theory is then empirically adequate if and only if the set $\Pi_{m} \cup \Gamma \cup\left\{\left(\Phi_{\mathfrak{A}}^{\left(E_{\mathfrak{A}}\right)}\right)^{o}: \mathfrak{A} \in \mathbf{A}\right\} \cup\left\{S^{o} E_{\mathfrak{A}}^{o}\right.$ : $\mathfrak{A} \in \mathbf{A}\}$ is semantically consistent for at least one $m \in M$. The use of semantic consistency is not incompatible with the RV, since it is essentially the same as the use of semantic entailment.

Corollary 2 is somewhat unsatisfying in that there is no difference between theoretical and observational concepts except their names. This is not a problem of the RV itself, but rather inherited from $\mathrm{CE}-$ which will become important in \$5. Nonetheless, there are some ways to provide the correspondence rules with a somewhat more elaborate role, even within the confines of CE. In keeping with Carnap's hope that all observational terms can be defined in theoretical terms and his almost universally accepted conjecture that the converse is not generally possible (Carnap 1966, 234), one can restrict the identification of theoretical with observational terms to observable objects. For the relation symbols, the correspondence rules then have the form

$$
\left\{\forall \bar{x}\left[P_{i}^{o} \bar{x} \leftrightarrow \exists Y\left(S Y \wedge Y x_{1} \wedge \cdots \wedge Y x_{m_{i}}\right) \wedge P_{i} \bar{x}\right]: i \in I\right\} \cup\left\{S^{o}=S\right\},
$$

where $m_{i}$ is the arity of $P_{i}$ and $\bar{x}$ stands for a string of $m_{i}$ distinct variables. ${ }^{7}$ Things are not as straightforward for functions, since functions have to be defined over the whole domain. One could thus substitute an $n_{j}+1$-ary observational relation $Q_{j}^{o}$ for each $n_{j}$-ary theoretical function $F_{j}$ with

$$
\forall \bar{x}\left[Q_{i}^{o} x_{1} \ldots x_{n_{j}+1} \leftrightarrow \exists Y\left(S Y \wedge Y x_{1} \wedge \cdots \wedge Y x_{n_{j}}\right) \wedge F_{j} x_{1} \ldots x_{n_{j}}=x_{n_{j}+1}\right],
$$

${ }^{7}$ To the extent that his terse sketch allows a comparison, Friedman (1982, 276-277) seems to have this kind of correspondence rule in mind. 
which would also mean that the descriptions $\left(\Phi_{\mathfrak{A}}^{\left(E_{\mathfrak{A}}\right)}\right)^{o}$ of the appearances have to be changed accordingly. It is therefore more convenient to disappoint Carnap's hope for explicit definitions and rely for each $F_{j}$ on the conditional definition

$$
\forall \bar{x}\left[\exists Y\left(S Y \wedge Y x_{1} \wedge \cdots \wedge Y x_{n_{j}}\right) \rightarrow\left(F_{j}^{o} \bar{x}=F_{j} \bar{x}\right)\right] .
$$

However, since the value of the observational functions is irrelevant outside the domains of the empirical substructures, the conditional definitions could be completed by conditionally defining that outside of the empirical subdomains, the observational functions all have the same, arbitrarily chosen value.

For reasons that will be discussed in $\$ 5$, observational constant symbols still have to be identified with their theoretical counterparts, which leads to

Corollary 3. In corollary 2, $\Gamma$ can also be the union of sets (3), (5), and $\left\{c_{k}^{o}=c_{k}\right.$ : $k \in K\}$.

Proof. Since the modification does not change the extensions of the observational terms within any set that is in the extension of $S$, the corollary follows immediately from corollary 2.

This, then, shows that van Fraassen's and Turney's conjecture about the impossibility of capturing empirical adequacy in the Rv is incorrect. The use of embeddings is thus not superior to the use of correspondence rules when connecting theory and observations. In fact, the CE-formalism is but a special case of the RV-formalism, with specific restrictions on the form of observation sentences, correspondence rules $\Gamma$, and postulates $\Pi$. In the next section, I will argue that because of these restriction, the CE-formalism is actually inferior to the Rv-formalism.

\section{Beyond empirical substructures}

Definition 3 puts enormous restrictions on the formalization of theories. For it follows from the definition of a substructure that every constant of a model $\mathfrak{T}_{n}$ of a CE-theory has to be in the domain $E$ of each of its empirical substructures $\mathfrak{E} \in \mathbf{E}_{n}$. Furthermore, every function of the model $\mathfrak{E} \in \mathbf{E}_{n}$ must map all (tuples of) elements of $E$ to elements of $E$ (Hodges 1993, lemma 1.2.2). If now a CE-theory $\left\langle\mathfrak{T}_{n}, \mathbf{E}_{n}\right\rangle$ is empirically adequate, every appearance $\mathfrak{A}$ is isomorphic to some empirical substructure of some $\mathfrak{T}_{n}$, and since $\left\{\mathfrak{T}_{n}\right\}_{n \in N}$ is closed under isomorphism, $\mathfrak{A}$ is an empirical substructure of some $\mathfrak{T}_{n}$ (Hodges 1993, ex. 1.2.4b). Therefore $\mathfrak{T}_{n}$ 's domain $T_{n}$ contains observable objects, all constants of $\mathfrak{T}_{n}$ are observable objects, and all functions of $\mathfrak{T}_{n}$ map observable objects to other observable objects. If now the theory is about, say, elementary particles, the observable objects are, for example, the results shown on the measurement instruments or (parts of) the measurement instruments themselves. These then 
have to satisfy those formulas that the theory ascribes to elementary particles, and all constants in the theory have to be results shown on measurement instruments or, respectively, (parts of) measurement instruments. ${ }^{8}$

One may object to these criticisms on the grounds that the terms 'embedding', 'substructure', and 'isomorphism' are not meant literally. However, the terms are well-defined within, but not outside of model theory, where in general they also do not occur together. And the objection makes van Fraassen use these terms in different, undefined ways without pointing this out, and subsequently helping himself to results from model theory without any justification. For example, van Fraassen $(1980,43)$ discusses cases in which "every model of $T_{1}$ can be embedded in (identified with a substructure of) a model of $T_{2}$." The parenthetical equivalence claim relies on the model theoretic definition of 'embedding' and 'substructure', on the closure of the class of models of a CE-theory under isomorphism, and the equivalence of embedding and the substructure relation for isomorphically closed classes of structures (Hodges 1993, ex. 1.2.4b). If the terms were not meant in the model theoretic sense, there would be no justification for this equivalence claim.

The problems resulting from van Fraassen's reliance on substructures can easily be solved in the RV, because RV-theories can contain non-observational terms, which themselves do not have to be connected directly to observations. Thus even without loosening the restrictions on the observation sentences inherited from $\mathrm{CE}$, the RV allows for connections between arbitrarily complex theoretical concepts with terms in $\mathscr{T}$ and simple observational concepts with terms in $\mathscr{O}$, namely through postulates $\Pi$ that introduce $\mathscr{T}$-terms and correspondence rules $\Gamma$ that go beyond simple identification or identification in a restricted domain. Hence in an RV-theory, there can be constant symbols in $\mathscr{T}$ for unobservable objects, and function symbols in $\mathscr{T}$ for functions from observable to unobservable objects. The empirical adequacy of such a more general Rv-theory $\Pi \cup \Gamma$ can still be defined as the semantic consistency of the set (2) of sentences. This solution in the RV no doubt has a counterpart in model theory, but it would in all likelihood either require either a significant reformulation of most scientific theories before they can be analyzed in the CE-formalism, or involve a generalization of the CEformalism. ${ }^{9}$ In the latter case the formalism would not be the one criticized here and conjectured by van Fraassen to be superior to the Rv-formalism.

\footnotetext{
${ }^{8}$ Essentially because of the Newman objection, this can formally always be arranged by defining appropriate relations, functions, and constants for the observable objects (assuming the right cardinality of the empirical substructures' and the appearances' domains), but this would trivialize empirical adequacy (cf. van Fraassen 2006); I am assuming here that the trivialization problem has been solved.

${ }^{9}$ Indeed, I think substituting relativized reducts for substructures in definition 5 suffices for avoiding the objections presented here.
} 


\section{Beyond consistency}

Corollary 2 allows a direct comparison of the RV and CE. Its relevance is demonstrated by a previous comparison given by $\operatorname{Muller}(2010,90)$, who, in line with Carnap (1966, 240-241), states that one aim of scientific theories in the RV is observational adequacy ("ObsAdeq"), for which a theory must entail the observations made so far. He points out three differences to empirical adequacy ("EmpAdeq") (Muller 2010, 90, n. 8):

(i) ObsAdeq relies on the distinction between theoretical and observational concepts whereas EmpAdeq does not [...]; (ii) ObsAdeq depends on historical time whereas EmpAdeq is timeless (quantifies universally over historical times); (iii) EmpAdeq requires for its definition a conception of a scientific theory that $[\ldots]$ cannot be defined in the $[\mathrm{RV}]$.

Corollary 2 shows that Muller's claim (iii), which is again but a conjecture, is false. It also shows that difference (i) is easily overcome by introducing an observational vocabulary and identity statements as correspondence rules. The discussion in $\$_{5}$ furthermore shows that this difference speaks in favor of the RV. Difference (ii) is not one of principle, but at best one of primacy of definition: One could define 'preliminary empirical adequacy' as 'empirical adequacy given the appearances A observed so far', and define 'eternal observational adequacy' as 'observational adequacy at all times'.

Muller misses the real difference, which is obvious in the syntactic paraphrase: A theory is observationally adequate if it entails all observations, while it is empirically adequate if it is not incompatible with them. Compared to observational adequacy, then, empirical significance is cheap: A tautology is compatible with any set of sentences describing the appearances, and thus its set of models is empirically adequate. This is obvious given claim 1, and can also be translated into the CE-formalism: The CE-theory corresponding to a tautology contains the class of all structures and for each structure the set of all its substructures as empirical substructures. This CE-theory is empirically adequate given any appearances A. Thus claim 1 throws doubt on van Fraassen's claim that science "aims to give us theories that are empirically adequate" (van Fraassen 1980, 12), at least if empirical adequacy is meant to be the only aim of science, or even a particularly interesting one.

This criticism has already been put forth by Worrall $\left(1984, \$_{3}\right)$ and Percival $(2007,87)$, who suggests as one of three possible replacements for van Fraassen's account of the aim of science that for each context $c$, science aims for theories that are empirically adequate and "fully empirically informative with respect to all the phenomena that are relevant at $c$ " (Percival 2007, 87)..$^{\circ}$ Unfortunately, Percival does not provide an explication of the latter notion in the CE-formalism,

\footnotetext{
${ }^{10}$ The other two possible replacements are outside the scope of this discussion.
} 
but rather stipulates that a theory "is 'fully empirically informative' with respect to certain phenomena iff it expresses all true propositions that are solely about those phenomena" (Percival 2007, n. 44). In his reply, van Fraassen $(2007, \mathbb{\$ 1 . 1}$ ) acknowledges the need for informative theories, but does not give an explication either.

To arrive at a precise notion of full empirical informativeness, one can look to the RV. An RV-theory $\Theta$ expresses all and only true propositions about some appearances $\mathbf{A}$ if and only if $\Theta \vDash\left\{\Phi_{\mathfrak{A}}^{\left(E_{\mathfrak{A}}\right)}: \mathfrak{A} \in \mathbf{A}\right\} \cup\left\{S E_{\mathfrak{A}}: \mathfrak{A} \in \mathbf{A}\right\}$. For a CE-theory $\left\langle\mathfrak{T}_{n}, \mathbf{E}_{n}\right\rangle$, then, every model $\mathfrak{T}_{n}^{*}$ of the corresponding family described by $\Theta$ is also a model of the observations. It is not very difficult to see that this is the case if and only if every empirical substructure $\mathfrak{E} \in \mathbf{E}_{n}$ of every structure in $\left\langle\mathfrak{T}_{n}, \mathbf{E}_{n}\right\rangle$ is isomorphic to an appearance $\mathfrak{A} \in \mathrm{A}$ and, since every $\mathfrak{A} \in \mathrm{A}$ is described up to isomorphism, vice versa. Since this condition already entails empirical adequacy, it formalizes the aim of science as suggested by Percival if context $c$ is taken to determine the appearances $\mathbf{A}$.

\section{Conclusion}

The preceding discussion has brought out a number of points. First, the RV can describe scientific theories and their relation to the the observations if $\mathrm{CE}$ can, while the opposite is not obviously the case. Second, the CE-formalism can and should be extended in at least two respects. It should allow for concepts in theories that do not also occur in the appearances and it should include the explication of 'full empirical informativeness' among its formal desiderata for a CE-theory. Finally, and as a suggestive corollary to this and previous discussions, the philosophy of science would be better off if syntactic and semantic methods in general and the $\mathrm{RV}$ and $\mathrm{CE}$ in particular were not seen as in opposition, but, correctly, as means of mutual support and improvement.

\section{References}

Bell, J. L. and Machover, M. (1977). A Course in Mathematical Logic. NorthHolland, Amsterdam.

Carnap, R. (1963). Replies and systematic expositions. In Schilpp, P. A., editor, The Philosophy of Rudolf Carnap, volume 11 of The Library of Living Philosophers, pages 859-1016. Open Court Publishing Company, Chicago and LaSalle, IL.

Carnap, R. (1966). Philosophical Foundations of Physics: An Introduction to the Philosophy of Science. Basic Books, Inc., New York and London. Edited by Martin Gardner. 
Carus, A. W. (2007). Carnap and Twentieth-Century Thought. Explication as Enlightenment. Cambridge University Press, Cambridge.

Chang, C. C. and Keisler, H. J. (1990). Model Theory, volume 73 of Studies in Logic and the Foundations of Mathematics. North Holland, Amsterdam, $3^{\text {rd }}$ edition. $3^{\text {rd }}$ impression 1992.

Enderton, H. B. (2009). Second-order and higher-order logic. In Zalta, E. N., editor, The Stanford Encyclopedia of Philosophy. The Metaphysics Research Lab, Center for the Study of Language and Information, Stanford University, spring 2009 edition.

Friedman, M. (1982). Review. The Journal of Philosophy, 79(5):274-283. Review of (van Fraassen 1980).

Halvorson, H. (2012). What scientific theories could not be. Philosophy of Sciences, 79(2):183-206.

Halvorson, H. (2013). The semantic view, if plausible, is syntactic. Philosophy of Science, $80(3): 475-478$.

Hodges, W. (1993). Model Theory, volume 42 of Encyclopedia of Mathematics and its Applications. Cambridge University Press, Cambridge. Digitally printed in 2008.

Justus, J. (2012). Carnap on concept determination: Methodology for philosophy of science. European Journal for Philosophy of Science, 2(2):161-179. Special issue: The Future of Philosophy of Science, edited by Stephan Hartmann and Jan Sprenger.

Lutz, S. (2009). Ideal language philosophy and experiments on intuitions. Studia Philosophica Estonica, 2.2:117-139. Special issue: The Role of Intuitions in Philosophical Methodology, edited by Daniel Cohnitz and Sören Häggqvist. http://www.spe.ut.ee/ojs-2.2.2/index.php/spe/article/view/65.

Lutz, S. (2012). On a straw man in the philosophy of science: A defense of the Received View. HOPOS: The Journal of the International Society for the History of Philosophy of Science, 2(1):77-120.

Lutz, S. (2014). What's right with a syntactic approach to theories and models? Erkenntnis. Forthcoming. Preprint: http://philsci-archive.pitt.edu/ archive/00005264/.

Maher, P. (2007). Explication defended. Studia Logica, 86(2):331-341.

Monton, B., editor (2007). Images of Empiricism. Essays on Science and Stances, with a Reply from Bas C. van Fraassen. Mind Association Occasional Series. Oxford University Press, Oxford. 
Monton, B. and Mohler, C. (2008). Constructive empiricism. In Zalta, E. N., editor, The Stanford Encyclopedia of Philosophy. The Metaphysics Research Lab, Center for the Study of Language and Information, Stanford University, Stanford, winter 2008 edition.

Mormann, T. (2007). The structure of scientific theories in logical empiricism. In Richardson and Uebel (2007), pages 136-162.

Muller, F. A. (2010). Reflections on the revolution at Stanford. Synthese, 183(1):87-114. Special issue: The Classical Model of Science II: The Axiomatic Method, the Order of Concepts and the Hierarchy of Sciences, edited by Arianne Betti, Willem de Jong and Marije Martijn.

Percival, P. (2007). An empiricist critique of constructive empiricism: The aim of science. In Monton (2007), chapter 6.

Richardson, A. (2007). "That sort of everyday image of logical positivism"Thomas Kuhn and the decline of logical empiricist philosophy of science. In Richardson and Uebel (2007), pages 346-370.

Richardson, A. and Uebel, T., editors (2007). The Cambridge Companion to Logical Empiricism. Cambridge University Press, Cambridge.

Turney, P. (1990). Embeddability, syntax, and semantics in accounts of scientific theories. Journal of Philosophical Logic, 19:429-451.

van Fraassen, B. C. (1970). On the extension of Beth's semantics of physical theories. Philosophy of Science, 37(3):325-339.

van Fraassen, B. C. (1980). The Scientific Image. The Clarendon Library of Logic and Philosophy. Clarendon Press, Oxford.

van Fraassen, B. C. (1989). Lawes and Symmetry. The Clarendon Library of Logic and Philosophy. Clarendon Press, Oxford.

van Fraassen, B. C. (1991). Quantum Mechanics: An Empiricist View. Clarendon Press, Oxford.

van Fraassen, B. C. (2002). The Empirical Stance. The Terry Lectures. Yale University Press, New Haven, CT.

van Fraassen, B. C. (2006). Representation: The problem for structuralism. Philosophy of Science, 73:536-547.

van Fraassen, B. C. (2007). From a view of science to a new empiricism. In Monton (2007), chapter 15 .

van Fraassen, B. C. (2008). Scientific Representation: Paradoxes of Perspective. Clarendon Press, Oxford. 
Worrall, J. (1984). An unreal image. The British Journal for the Philosophy of Science, 35:65-80. Review of (van Fraassen 1980). 